\title{
Distribution of streaming rates into high-redshift galaxies
}

\author{
Tobias Goerdt, ${ }^{1 \star}$ Daniel Ceverino, ${ }^{2,3}$ Avishai Dekel ${ }^{4}$ and Romain Teyssier ${ }^{5}$ \\ ${ }^{1}$ Institut für Astrophysik, Universität Wien, Türkenschanzstraße 17, A-1180 Wien, Austria \\ ${ }^{2}$ Centro de Astrobiología (CSIC-INTA), Ctra de Torrejón a Ajalvir, km 4, E-28850 Torrejón de Ardoz, Madrid, Spain \\ ${ }^{3}$ Astro-UAM, Universidad Autónoma de Madrid, Unidad Asociada CSIC, E-28049 Madrid, Spain \\ ${ }^{4}$ Racah Institute of Physics, The Hebrew University, Jerusalem 91904, Israel \\ ${ }^{5}$ Institute for Computational Science, Universität Zürich, Winterthurer Strasse 190, CH-8057 Zürich, Switzerland
}

Accepted 2015 August 27. Received 2015 July 30; in original form 2015 April 28

\begin{abstract}
We study the accretion along streams from the cosmic web into high-redshift massive galaxies using three sets of AMR hydrocosmological simulations. We find that the streams keep a roughly constant accretion rate as they penetrate into the halo centre. The mean accretion rate follows the mass and redshift dependence predicted for haloes by the EPS approximation, $\dot{M} \propto M_{\text {vir }}^{1.25}(1+z)^{2.5}$. The distribution of the accretion rates can well be described by a sum of two Gaussians, the primary corresponding to 'smooth inflow' and the secondary to 'mergers'. The same functional form was already found for the distributions of specific star formation rates in observations. The mass fraction in the smooth component is $60-90$ per cent, insensitive to redshift or halo mass. The simulations with strong feedback show clear signs of reaccretion due to recycling of galactic winds. The mean accretion rate for the mergers is a factor 2-3 larger than that of the smooth component. The standard deviation of the merger accretion rate is $0.2-0.3 \mathrm{dex}$, showing no trend with mass or redshift. For the smooth component it is $0.12-0.24$ dex.
\end{abstract}

Key words: methods: numerical-galaxies: evolution-galaxies: formation-galaxies: high redshift-intergalactic medium - cosmology: theory.

\section{INTRODUCTION}

The dominant idea of galaxy formation has changed recently. Decades ago, it was thought (Rees \& Ostriker 1977; Silk 1977; White \& Rees 1978) that galaxies collect their baryons through diffuse gas symmetrically falling into dark matter haloes and being shock-heated as it hits the gas residing in them - "hot mode accretion'. The mass of the halo decides if the gas will eventually settle into the galaxy. It was shown by theoretical work and simulations (Fardal et al. 2001; Birnboim \& Dekel 2003; Kereš et al. 2005, 2009; Dekel \& Birnboim 2006; Ocvirk, Pichon \& Teyssier 2008; Dekel et al. 2009, 2013) that galaxies at high redshift $(z \gtrsim 2)$, acquire their baryons primarily via cold streams of relatively dense and pristine gas with temperatures around $10^{4} \mathrm{~K}$ that penetrate through the diffuse shock-heated medium - 'cold mode accretion'. The peak of the stream activity is around redshift 3 . Theoretical work predicted a quenching of the gas supply into high-mass galaxies $\left(M_{\mathrm{vir}}>\right.$ $\left.10^{12} \mathrm{M}_{\odot}\right)$ at low redshifts $(z<2$; Dekel \& Birnboim 2006).

Genel et al. (2010) showed with $N$-body simulations that about half of the dark-matter haloes' mass is built up smoothly. So as the galaxies grow baryons should also be accreted semicontinuously.
Indeed Dekel et al. (2009) showed with hydrodynamical cosmological simulations that about two-thirds of the mass are brought in by the rather smooth gas components, that also consist of miniminor mergers with mass ratio smaller than 1:10. The smooth and steady accretion through the cold streams may have been the main driver of the formation of the massive, clumpy and star-forming discs that have been observed at $z \sim 2$ (Genel et al. 2008; Genzel et al. 2008; Förster Schreiber et al. 2009, 2011). Major merger events on the other hand may have only contributed the smaller part (Ceverino, Dekel \& Bournaud 2010; Agertz, Teyssier \& Moore 2011; Ceverino et al. 2015).

The times scales for the gas depletion of the galaxies are relatively short compared to the Hubble time during all of cosmic history (Daddi et al. 2010; Genzel et al. 2010). So galaxies must always accrete fresh gas coming from the intergalactic medium in order to sustain star formation over such a long time at the observed level. The system is self-regulated: the accretion is setting the star formation rate independent of the amount of available gas (Bouché et al. 2010). The galaxy's star formation rates are fundamentally limited by the gas inflow. Local physics like the regulation by feedback seem to be only of secondary importance. A fact that highlights the accretion rates' importance: the time-scales for star formation rate and star formation both crucially depend on the gas accretion rate. A robust approximation for the average growth rate 
of halo virial mass $M_{\mathrm{vir}}$ has been derived (Neistein, van den Bosch \& Dekel 2006; Neistein \& Dekel 2008) using the EPS (Lacey \& Cole 1993) theory of cosmological clustering into spherical haloes in virial equilibrium.

Attempts to prove the cold accretion stream paradigm observationally are ongoing: the characteristics of $\operatorname{Ly} \alpha$ emission produced by the cold gas streams via the release of gravitational energy ${ }^{1}$ have been predicted using cosmological hydrodynamical AMR simulations (Goerdt et al. 2010). The simulated Ly $\alpha$ blobs (LABs) can reproduce many of the features of the observed LABs. They can therefore be interpreted as direct observational detections of cold stream accretion. Likelihoods of observing streams in absorption have been theoretically predicted (Goerdt et al. 2012). A planar subgroup of satellites has been found to exist in the Andromeda galaxy (M 31; Ibata et al. 2013). It comprises about half of the population. This vast thin disc of satellites is a natural result of cold stream accretion (Goerdt, Burkert \& Ceverino 2013) and can therefore also be treated as observational evidence for the existence of cold streams.

Observationally, two main modes of star formation are known to control the growth of galaxies: a relatively steady one in disclike galaxies and a starburst mode which is generally interpreted as driven by merging. Rodighiero et al. (2011) quantified the relative contribution of the two modes in the redshift interval $1.5<z<2.5$ using PACS/Herschel observations over the whole COSMOS and GOODS-South fields, in conjunction with previous optical/nearinfrared data. Sargent et al. (2012) used their data to predict the shape of the infrared luminosity function at redshifts $z \leq 2$ by introducing a double-Gaussian decomposition of the specific star formation rate distribution at fixed stellar mass into a contribution (assumed redshift- and mass invariant) from main-sequence and starburst activity.

The characteristics of baryonic accretion on to galaxies have been analysed with the help of cosmological hydrodynamic simulations in various works. Key results are that gas accretion is mostly smooth, with mergers only becoming important for groups and clusters. The specific rate of the gas accretion on to haloes is only weakly dependent on the halo mass (van de Voort et al. 2011). The accreted gas is bimodal with a temperature division at $10^{5} \mathrm{~K}$. Cold-mode accretion dominates inflows at early times and declines below $z \sim 2$. Hot-mode accretion peaks near $z=1-2$ and declines gradually. Cold-mode accretion can fuel immediate star formation, while hot-mode accretion preferentially builds a large, hot gas reservoir in the halo (Woods et al. 2014). Another third distinct accretion mode along with the 'cold' and 'hot' modes has been identified (Oppenheimer et al. 2010), called 'recycled wind mode', in which the accretion comes from material that was previously ejected from a galaxy. Galaxies in substantially overdense environments grow predominantly by a smooth accretion from cosmological filaments which dominates the mass input from mergers (Romano-Díaz et al. 2014). The relationship between stellar mass, metallicity and star formation rate can be explained by metal-poor gas accretion (Almeida et al. 2014).

The properties of cold- and hot-mode gas, are clearly distinguishable in the outer parts of massive haloes. The cold-mode gas is confined to clumpy filaments that are approximately in pressure equilibrium with the hot-mode gas. Cold-mode gas typically has a much lower metallicity and is much more likely to be infalling

\footnotetext{
${ }^{1}$ The gas remains at constant velocity as it flows down the potential gradient towards the halo centre (Goerdt \& Ceverino 2015).
}

(van de Voort \& Schaye 2012). Stream morphologies become increasingly complex at higher resolution, with large coherent flows revealing density and temperature structure at progressively smaller scales (Nelson et al. 2015).

The cold gas accretion rate is not a universal factor of the dark matter accretion rate. Galactic winds can cause star formation rates to deviate significantly from the external gas accretion rates, both via gas ejection and reaccretion. Cold accretion is broadly consistent with driving the bulk of the highly star-forming galaxies observed at $z \sim 2$ (Faucher-Giguère, Keres \& Ma 2011). The key physical variables of galactic cold inflows substantially decrease with decreasing redshift, namely: the number of streams, the average inflow rate per stream as well as the mean gas density in the streams. The stream angular momentum on the other hand is found to increase with decreasing redshift (Cen 2014).

The average radial velocities of the inflowing material decrease with decreasing radius (roughly a decrease of 65 per cent between $1 r_{\text {vir }}$ and the centre of the host; Wetzel \& Nagai 2015, their figs 4 and 6 , bottom panels). The velocity profile of the gas flowing along the streams into a galaxy's halo in the form of cold streams is, contrary to what might be expected, roughly constant with radius instead of free-falling (Goerdt \& Ceverino 2015).

In a forthcoming companion paper (Goerdt et al., in preparation), we are going to address the role of mergers versus smooth flows by analysing the clumpiness of the gas streams. We will evaluate each clump mass and estimate a mass ratio for the expected merger. Finally, we are going to look at the distribution of constituents amongst the clumps.

In this paper, we look at the amount of inflow - the mass accretion rate - as a function of radius, mass and redshift for the three constituents gas, stars and dark matter. The paper is organized as follows: in Section 2, we present the three suites of simulations used for the analysis. In Section 3, we show individual and averaged inflow curves. In Section 4, we look at the distributions of the amount of inflow, i.e. at the deviations from the means presented in Section 3. In Section 5, we draw our conclusions.

\section{SIMULATIONS}

Galaxy snapshots of three different suites of cosmological simulations based on Eulerian AMR (Adaptive Mesh Refinement) hydrodynamics are analysed for this paper. The three suites are the ADAPTIVE REFINEMENT TREE (hereafter ART; Ceverino et al. 2010, 2012; Dekel et al. 2013; Ceverino et al. 2015), the $A_{\mathrm{RP}}$ (Ceverino et al. 2014; Zolotov et al. 2015) as well as the HorizonMareNostrum (hereafter MN; Ocvirk et al. 2008) simulation. The ART and the $A_{\mathrm{RP}}$ suites of simulations both consist of several simulations zooming in with a maximum resolution of $15-70 \mathrm{pc}$ at $z=2$ on individual galaxies that reside in dark matter haloes of masses $(0.07-1.98) \times 10^{12} \mathrm{M}_{\odot}$ at $z=2.3$. The $\mathrm{MN}$ simulation contains hundreds of massive galaxies in a cosmological box of side $71 \mathrm{Mpc}$ with a maximum resolution of $\simeq 1 \mathrm{kpc}$.

To show the morphology of the gas streams we present in Fig. 1 gas influx maps of two sample galaxies from the MN and the ART suite. The panels demonstrate the dominance of typically three, narrow cold streams, which come from well outside the virial radius along the dark-matter filaments of the cosmic web, and penetrate into the discs at the halo centres. The streams are partly clumpy and partly smooth, even in the simulations with higher resolution. 

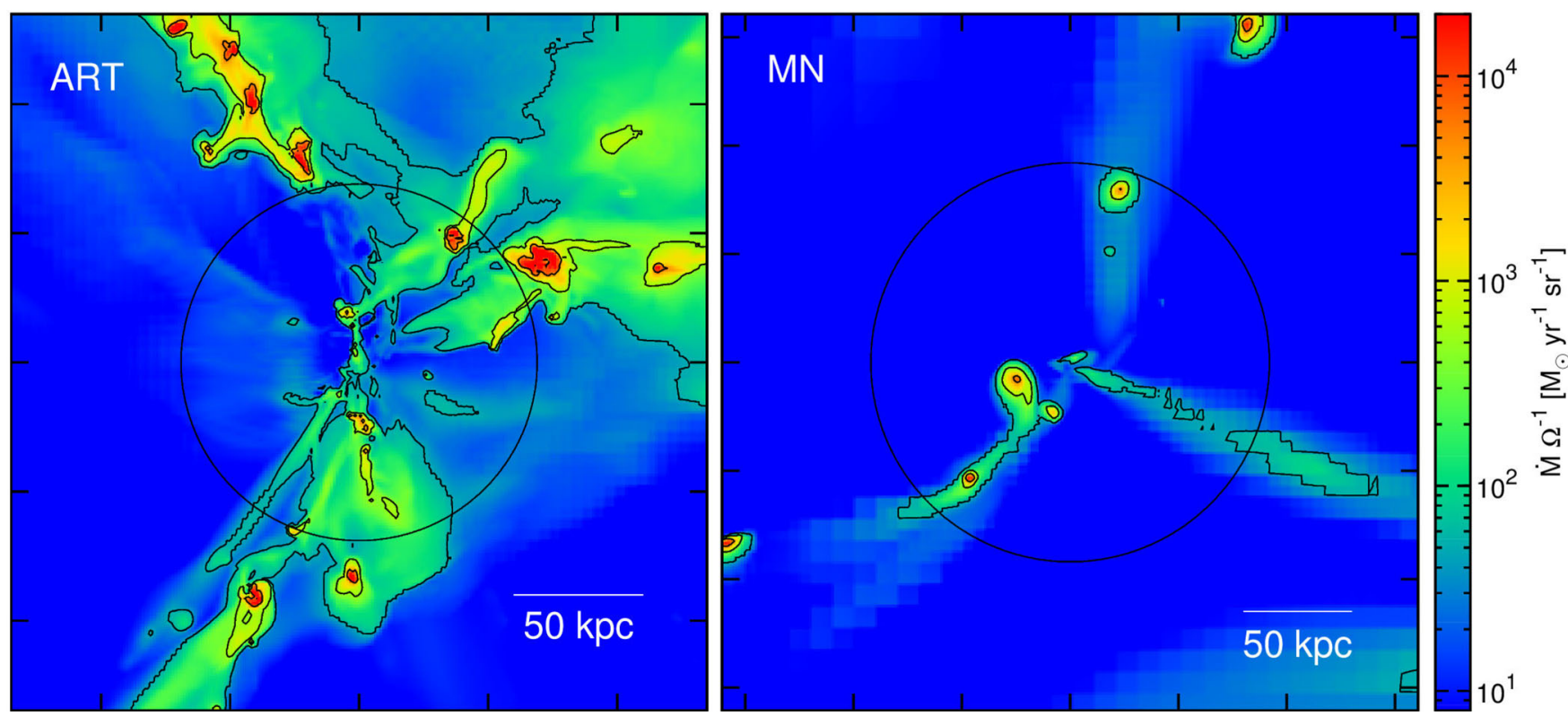

Figure 1. Simulated galaxies from two different suites of simulations (MN and ART). Shown in colour coding is the peak gas inflow along the line of sight. Inflow is here defined as the radial gas inflow per unit solid angle. The contours indicate gas influxes of $\dot{M} \Omega^{-1}=50,500$ and $5000 \mathrm{M}_{\odot} \mathrm{yr}^{-1} \mathrm{sr}^{-1}$, respectively. The circles refer to the virial radii. Left: one of the ART galaxies (resolution $70 \mathrm{pc}$ ) at $z=2.3$, with $M_{\mathrm{vir}}=3.5 \times 10^{11} \mathrm{M}_{\odot}$. Right: a typical MN galaxy (resolution $1 \mathrm{kpc}$ ) at $z=2.5$, with $M_{\mathrm{vir}}=10^{12} \mathrm{M}_{\odot}$. The inflow is dominated in both cases by three cold narrow streams that are partly clumpy.

\subsection{High-resolution ART simulations}

The ART simulations were run with the AMR code ART (Kravtsov, Klypin \& Khokhlov 1997; Kravtsov 2003) with a spatial resolution better than $70 \mathrm{pc}$ in physical units. It incorporates the relevant physical processes for galaxy formation that are: gas cooling, photoionization heating, star formation, metal enrichment and stellar feedback (Ceverino \& Klypin 2009). Cooling rates were computed for the given gas density, temperature, metallicity and UV background based on CLOUDY (Ferland et al. 1998). Cooling is assumed at the centre of a cloud of thickness $1 \mathrm{kpc}$ (Ceverino-Rodriguez 2008; Robertson \& Kravtsov 2008). Metallicity-dependent, metalline cooling is included, assuming a relative abundance of elements equal to the solar composition. The code implements a 'constant' feedback model, in which the combined energy from stellar winds and supernova ( $\mathrm{SN}$ ) explosions is released as a constant heating rate over $40 \mathrm{Myr}$ (the typical age of the lightest star that can still explode in a Type II supernova). Photoheating is also taken into account self-consistently with radiative cooling. A uniform UV background based on the Haardt \& Madau (1996) model is assumed. Local sources are ignored. In order to mimic the selfshielding of dense, galactic neutral hydrogen from the cosmological UV background, the simulation assumes for the gas at total densities above $n=0.1 \mathrm{~cm}^{-3}$ a substantially suppressed UV background $\left(5.9 \times 10^{26} \mathrm{erg} \mathrm{s}^{-1} \mathrm{~cm}^{-2} \mathrm{~Hz}^{-1}\right.$, the value of the pre-reionization $\mathrm{UV}$ background at $z=8$ ). The self-shielding threshold of $n=0.1 \mathrm{~cm}^{-3}$ is set by radiative transfer simulations of ionizing photons coming from the cosmological background as well as from the galaxy itself (see appendix A2 of Fumagalli et al. 2011).

The ART code has a unique feature for the purpose of simulating the detailed structure of the streams. It allows gas cooling to well below $10^{4} \mathrm{~K}$. This enables high densities in pressure equilibrium with the hotter and more dilute medium. A non-thermal pressure floor has been implemented to ensure that the Jeans length is resolved by at least seven resolution elements and thus prevent artificial fragmentation on the smallest grid scale (Truelove et al. 1997; Robertson \& Kravtsov 2008; Ceverino et al. 2010). It is effective in the dense $\left(n>10 \mathrm{~cm}^{-3}\right)$ and cold $\left(T<10^{4} \mathrm{~K}\right)$ regions inside galactic discs.

The equation of state remains unchanged at all densities. Stars form in cells where the gas temperature is below $10^{4} \mathrm{~K}$ and the gas density is above a threshold of $n=1 \mathrm{~cm}^{-3}$ according to a stochastic model that is consistent with the Kennicutt (1998) law. In the creation of a single stellar particle, a constant fraction (1/3) of the gas mass within a cell is converted into stellar mass (see appendix of Ceverino \& Klypin 2009). The interstellar medium (ISM) is enriched by metals from supernovae Type II and Type Ia. Metals are released from each star particle by SN II at a constant rate for $40 \mathrm{Myr}$ after its birth. A Miller \& Scalo (1979) initial mass function (IMF) is assumed which is matching the results of Woosley \& Weaver (1995). The metal ejection by SN Ia assumes an exponentially declining $\mathrm{SN}$ Ia rate from a maximum at $1 \mathrm{Gyr}$. The code treats the advection of metals self-consistently and it distinguishes between SN II and SN Ia ejecta (Ceverino-Rodriguez 2008).

The initial conditions for the ART simulations were created using low-resolution cosmological $\mathrm{N}$-body simulations in comoving boxes of side 29-114 Mpc. Its cosmological parameters were motivated by WMAP5 (Komatsu et al. 2009). The values are: $\Omega_{\mathrm{m}}=0.27$, $\Omega_{\Lambda}=0.73, \Omega_{\mathrm{b}}=0.045, h=0.7$ and $\sigma_{8}=0.82$. We selected 31 haloes of $M_{\mathrm{vir}} \simeq 10^{12} \mathrm{M}_{\odot}$ at $z=1.0$. For each halo, a concentric sphere of radius twice the virial radius was identified for resimulation with high resolution. Gas was added to the box following the dark matter distribution with a fraction $f_{\mathrm{b}}=0.15$. The whole box was then resimulated, with refined resolution in the selected volume about the respective galaxy. The dark matter particle mass is $5.5 \times 10^{5} \mathrm{M}_{\odot}$, the minimum star particle mass is $10^{4} \mathrm{M}_{\odot}$, the smallest cell size is $35 \mathrm{pc}$ (physical units) at $z=2$. 


\subsubsection{Simulations including radiation pressure $\left(\mathrm{A}_{\mathrm{RP}}\right)$}

The $A_{\mathrm{RP}}$ suite of simulations (Ceverino et al. 2014; Zolotov et al. 2015 ) is a further development of last subsection's ART suite: apart from the features already presented there, it also includes the effects of radiation pressure by massive stars. The radiation pressure was modelled as a non-thermal pressure that acts only in dense and optically thick star-forming regions in a way that the ionizing radiation injects momentum around massive stars, pressurizing star-forming regions (Agertz et al. 2013, their appendix B). The $A_{\mathrm{RP}}$ suite uses a stronger feedback that brings the stellar-to-halo mass ratio closer to the estimates by abundance matching (Behroozi, Wechsler \& Conroy 2013; Moster, Naab \& White 2013). See Ceverino et al. (2014) and Moody et al. (2014). The adaptive comoving mesh has been refined in the dense regions to cells of minimum size between 17 and $35 \mathrm{pc}$ in physical units. The DM particle mass is $8.3 \times 10^{4} \mathrm{M}_{\odot}$. The particles representing star clusters have a minimum mass of $10^{3} \mathrm{M}_{\odot}$, similar to the stellar mass of an Orion-like star cluster. The initial conditions for the $A_{\mathrm{RP}}$ simulations were created using low-resolution cosmological $N$-body simulations in comoving boxes of side $14-57 \mathrm{Mpc}$. We selected 34 haloes of $M_{\mathrm{vir}}=1.18-14.7 \times 10^{11} \mathrm{M}_{\odot}$ at $z=1.0$.

\subsection{RAMSES HORIZON-MARENOSTRUM SIMULATION}

The MN simulation uses the AMR code RAMSES (Teyssier 2002). The spatial resolution is $\sim 1 \mathrm{kpc}$ in physical units. UV heating is included assuming the Haardt \& Madau (1996) background model, as in the ART simulation. The code incorporates a simple model of supernovae feedback and metal enrichment using the implementation described in Dubois \& Teyssier (2008). The cooling rates are calculated assuming ionization equilibrium for $\mathrm{H}$ and $\mathrm{He}$, including both collisional ionization and photoionization (Katz, Hernquist \& Weinberg 1992). Metal cooling is also included using tabulated CLOUDY rates, and is assumed proportional to the metallicity, relative to the Grevesse \& Sauval (1998) solar abundances. Unlike in the ART simulation, no cooling below $T<10^{4} \mathrm{~K}$ is computed, and no self-shielding of the UV flux is assumed.

For high-density regions, the RAMSES code considers a polytropic equation of state with $\gamma_{0}=5 / 3$ to model the complex, multiphase and turbulent structure of the ISM (Yepes et al. 1997; Springel \& Hernquist 2003) in a simplified form (see Dubois \& Teyssier 2008; Schaye \& Dalla Vecchia 2008). The ISM is defined as gas with hydrogen density greater than $n_{\mathrm{H}}=0.1 \mathrm{~cm}^{-3}$, one order of magnitude lower than in the ART simulation. Star formation has been included, for ISM gas only, by spawning star particles at a rate consistent with the Kennicutt (1998) law derived from local observations of star-forming galaxies.

The MN simulation implemented a pressure floor in order to prevent artificial fragmentation, by keeping the Jeans lengthscale, $\lambda_{\mathrm{J}} \propto T n^{-2 / 3}$, larger than the size of four grid cells everywhere. At every position where $n>0.1 \mathrm{~cm}^{-3}$, a density dependent temperature floor was imposed. It mimics the average thermal and turbulent pressure of the multiphase ISM (Springel \& Hernquist 2003; Dalla Vecchia \& Schaye 2008). The gas is allowed to heat up above this temperature floor and cool back again. The temperature floor follows a polytropic equation of state with $T_{\text {floor }}=T_{0}\left(n / n_{0}\right)^{\gamma_{0}-1}$, where $T_{0}=10^{4} \mathrm{~K}$ and $n_{0}=0.1 \mathrm{~cm}^{-3}$. The resulting pressure floor is given by $P_{\text {floor }}=n_{\mathrm{H}} k_{\mathrm{B}} T_{\text {floor }}$.

For each stellar population, 10 percent of the mass is assumed to turn into supernovae Type II after $10 \mathrm{Myr}$, where the energy and metals are released in a single impulse. For each supernova,
Table 1. The various bins of galaxies for our analyses. They are used throughout the whole paper. 'Label' is the tag the corresponding bin is labelled by in our figures. It is a mass close to the ensemble's actual mean virial mass. 'Suite' denotes the suite of simulations the bin stems from. $M_{\mathrm{vir}}$ is the mean virial mass of the bin together with its standard deviation. $z$ is the mean redshift of the ensemble together with its standard deviation. Galaxies from different MN snapshots are not combined therefore the redshift's standard deviation is always zero for all MN bins. $N_{\text {gal }}$ gives the total number of galaxies in the given bin.

\begin{tabular}{rcccr}
\hline Label & Suite & $M_{\text {vir }}\left[10^{12} \mathrm{M}_{\odot}\right]$ & $z$ & $N_{\text {gal }}$ \\
\hline $10^{13}$ & MN & $10.47 \pm 0.56$ & 1.57 & 12 \\
$10^{13}$ & MN & $10.49 \pm 0.93$ & 2.46 & 12 \\
$5 \times 10^{12}$ & MN & $5.00 \pm 0.045$ & 1.57 & 12 \\
$5 \times 10^{12}$ & MN & $5.48 \pm 0.26$ & 2.46 & 11 \\
$10^{12}$ & MN & $1.03 \pm 0.003$ & 1.57 & 8 \\
$10^{12}$ & MN & $1.01 \pm 0.004$ & 2.46 & 12 \\
$10^{12}$ & MN & $1.03 \pm 0.006$ & 4.01 & 9 \\
$10^{11}$ & MN & $0.099 \pm 0.000$ & 1.57 & 12 \\
$10^{11}$ & MN & $0.099 \pm 0.000$ & 2.46 & 7 \\
$10^{11}$ & MN & $0.099 \pm 0.000$ & 4.01 & 12 \\
$1.9 \times 10^{12}$ & ART & $1.907 \pm 0.217$ & $1.14 \pm 0.02$ & 34 \\
$1.3 \times 10^{12}$ & ART & $1.286 \pm 0.093$ & $1.60 \pm 0.02$ & 73 \\
$8.6 \times 10^{11}$ & ART & $0.863 \pm 0.046$ & $2.25 \pm 0.02$ & 109 \\
$3.9 \times 10^{11}$ & ART & $0.391 \pm 0.034$ & $3.40 \pm 0.04$ & 119 \\
$7.1 \times 10^{11}$ & $A_{\text {RP }}$ & $0.707 \pm 0.055$ & $1.14 \pm 0.02$ & 41 \\
$6.7 \times 10^{11}$ & $A_{\text {RP }}$ & $0.672 \pm 0.049$ & $1.58 \pm 0.02$ & 47 \\
$4.9 \times 10^{11}$ & $A_{\text {RP }}$ & $0.491 \pm 0.028$ & $2.21 \pm 0.03$ & 57 \\
$2.9 \times 10^{11}$ & $A_{\text {RP }}$ & $0.290 \pm 0.019$ & $3.27 \pm 0.05$ & 62 \\
$2.6 \times 10^{11}$ & $A_{\text {RP }}$ & $0.260 \pm 0.010$ & $1.13 \pm 0.02$ & 40 \\
$2.4 \times 10^{11}$ & $A_{\text {RP }}$ & $0.242 \pm 0.010$ & $1.59 \pm 0.02$ & 50 \\
$1.6 \times 10^{11}$ & $A_{\text {RP }}$ & $0.160 \pm 0.007$ & $2.29 \pm 0.03$ & 60 \\
$7.3 \times 10^{10}$ & $A_{\text {RP }}$ & $0.073 \pm 0.004$ & $3.53 \pm 0.05$ & 66 \\
\hline
\end{tabular}

10 per cent of the ejected mass is assumed to be pure metals, with the remaining 90 per cent keeping the metallicity of the star at birth. $\mathrm{SN}$ Ia feedback has not been considered. The initial conditions of the $\mathrm{MN}$ simulation were constructed assuming a $\Lambda$ cold dark matter ( $\Lambda$ CDM) universe with $\Omega_{\mathrm{M}}=0.3, \Omega_{\Lambda}=0.7, \Omega_{\mathrm{b}}=0.045, h=0.7$ and $\sigma_{8}=0.9$ in a periodic box of $71 \mathrm{Mpc}$. The adaptive-resolution rules in this simulation were the same everywhere, with no zoom-in resimulation of individual galaxies. The dark matter particle mass is $1.16 \times 10^{7} \mathrm{M}_{\odot}$, the star particle mass is $2.05 \times 10^{6} \mathrm{M}_{\odot}$, the smallest cell size is $1.09 \mathrm{kpc}$ physical and the force softening length is $1.65 \mathrm{kpc}$.

We will always show averaged results for an ensemble of galaxies having very similar masses and redshifts. In Table 1, we show a summary of the various bins of galaxies we use. Since we have better statistics for the MN simulation we were able to bin galaxies from a narrower mass range; therefore, the standard deviations of the mean mass of an MN bin is usually much smaller. To partly compensate for that we combine ART galaxies from adjacent redshifts increasing the statistics but introducing a standard deviation into the mean redshift of the bin.

\section{AMOUNT OF INFLOW}

First, the average inflow is computed as a function of radius from the simulations. To do so, the amount of mass is measured that crosses within a small time $\Delta t$ a spherical shell of radius $r$ that is centred around any given host galaxy. This is done independently for gas, stars and dark matter, which are the three different constituents in the simulations. To get an inflow rate, we divide the mass that is crossing through a given shell by the time $\Delta t$ taken. The reader 
should note that we do include the expansion due to the Hubble flow in this calculation. As with most cosmological codes only the peculiar velocities are written out into the snapshots on to hard disc. We then add the velocity of the Hubble expansion as measured from the centre of the respective host halo by hand. The crucial difference between stars and dark matter particles on the one hand and gas on the other hand is that stars and dark matter are collisionless and therefore virialized within the virial radius $r_{\text {vir }}$, whereas gas on the other hand is neither. In case of the gas inflow we chose to only take into account inwards radial velocity cells in order to get the cleanest estimate for the amount of the inflow. Whereas for stars and dark matter we have to take into account all the material (i.e. only the net influx). If one takes into account only the inflowing stellar particles, the difference would not be very big, the stellar inflow rates would only be $\sim 25$ per cent bigger. The stars follow the gas very precisely. For the dark matter particles on the other hand the differences are tremendous: the inflow rates increase by more than a factor of 2 . It is worth stressing that we do not use any information about the temperature: so hot inflowing gas is taken into account, too. The hot gas however contributes very little to the inflow, since the vast majority of the mass of the material is in the cold phase.

We also want to briefly look at the inflow rates of all cells to compare those to the ones from our choice of taking into account only the cells which have an inwards radial velocity. The results vary for the different suites of simulations: for the MN suite, the one with the weakest feedback, there is hardly any difference: the inflow rates are still constant at roughly the same level. For the ART simulations which has stronger feedback implemented, the average inflow rate drops by a factor of two, when also taking into account cells with outflowing velocities. Also we see that the inflow rates are smaller towards the centre of the host halo. An effect already described by van de Voort \& Schaye (2012, when taking into account only inflowing gas, they find approximately constant accretion rates as well). For the $A_{\mathrm{RP}}$ suite of simulations which has by far the strongest feedback the average inflow rate drops by a factor of 10 , but for this suite the inflow rates are higher towards the centre of the host halo. We see an inverted van de Voort \& Schaye (2012) effect. The differences between taking outflowing gas cells into account or not might seem large, the reader however should note that we are mainly interested in the inflowing material and not so much the outflowing material, so concentrating on the inflowing cells only is the right choice.

We compute inflow rates for the galaxies. For reducing the statistical noise that is present in the inflow of a single galaxy we stack the amount of inflow of all available galaxies having similar redshifts and masses, from all three suites of simulations galaxies that are available at a variety of different halo masses and redshifts. Since the $\mathrm{MN}$ is a fully cosmological simulation, it is possible to compile bins of galaxies spanning several orders of magnitude in mass for various redshifts. The ART suite consists only of resimulated galaxies at comparable masses and therefore there is at any given redshift only one mass bin available which evolves in mass with cosmic time. The situation of the $A_{\mathrm{RP}}$ suite is similar, however for this suite, two different mass bins are available at the given redshifts, but those masses also evolve with cosmic time. The average halo masses and redshifts of the chosen bins are summarized in Table 1.

In Fig. 2, the average inflow as a function of radius for galaxies at $z=2.2-2.5$ with $M_{\text {vir }}=5-10 \times 10^{11} \mathrm{M}_{\odot}$ of all three $\mathrm{MN}$, ART and $A_{\mathrm{RP}}$ are shown. The galaxies have an average 'ground' inflow in gas of about $100 \mathrm{M}_{\odot} \mathrm{yr}^{-1}$ which is fairly constant over the different radii. This is the smooth component of the inflow, the cold streams. On top of this smooth ground inflow, there are

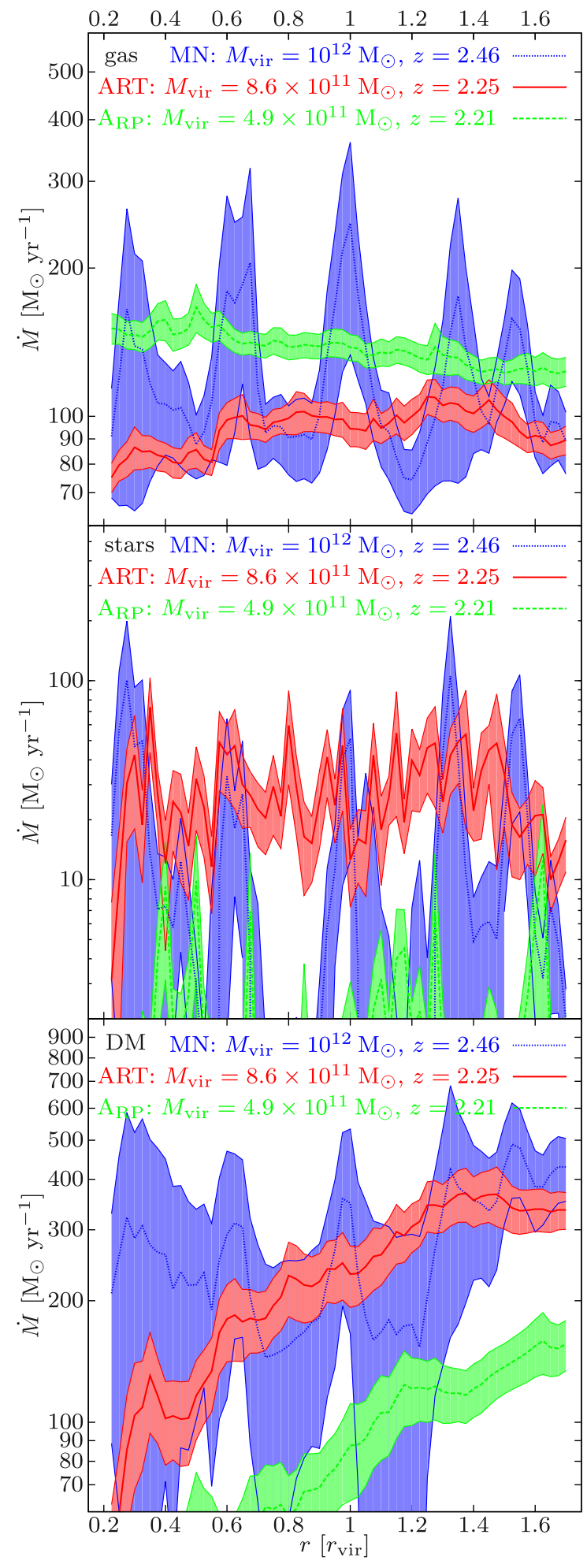

Figure 2. The average inflow as a function of radius together with its $1 \sigma$ standard deviations. The MN (dotted blue), the ART (solid red) and the $A_{\mathrm{RP}}$ (dashed green) simulations are compared. In gas (top panel) an average 'ground' inflow is seen in gas which is fairly constant over the different radii. On top of this smooth ground inflow there are distinct peaks in the inflow, corresponding to bigger clumps. In the stellar distribution (middle panel) signals of inflow are only seen at those gas peaks. Stars seem to form only within those gas clumps. The stellar inflow rates are noisy but roughly constant. In dark matter (bottom panel) the inflow increases with radius. The gas inflow of the $A_{\mathrm{RP}}$ simulation is fairly high due to its recycling processes. 


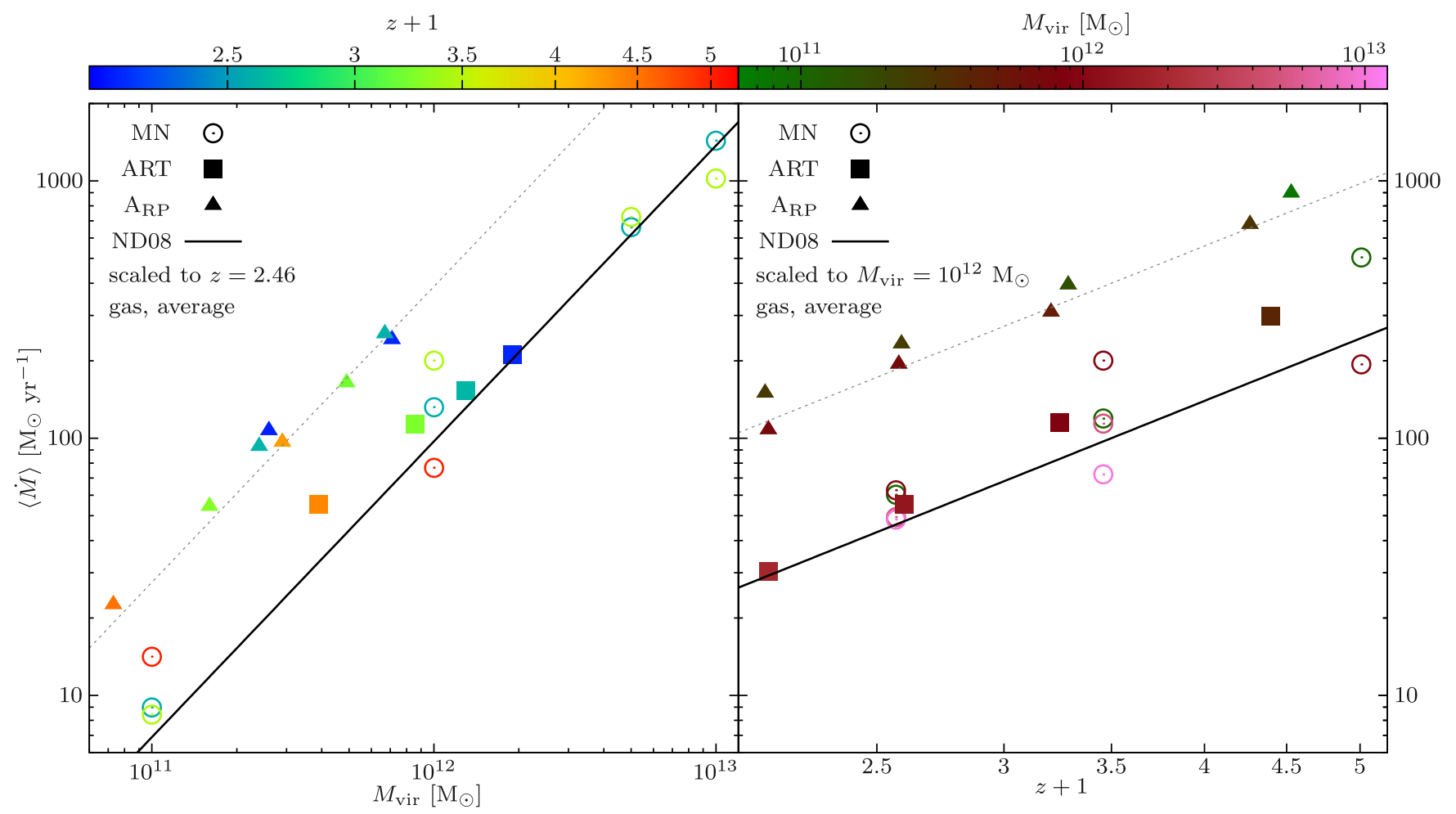

Figure 3. The amount of inflow $M_{\text {inflow }}$ of the gas as a function of halo mass $M_{\mathrm{vir}}$ (left-hand panel) or as a function of redshift (right-hand panel). The ART data is plotted as filled squares, the MN data as open circles and the $A_{\mathrm{RP}}$ data as filled triangles. The data points have been rescaled according to $\langle\dot{M}\rangle \propto[1+z]^{2.5}$ (left-hand panel) or $\langle\dot{M}\rangle \propto\left[M_{\mathrm{vir}}\right]^{1.15}$ (right-hand panel) in order to match $z=2.46$ (left-hand panel) or $M_{\mathrm{vir}}=10^{12} \mathrm{M}_{\odot}$ (right-hand panel). The original redshift or host halo mass values are given in colour coding. The $A_{\mathrm{RP}}$ values are higher than the other two simulations, but they also follow clearly the same slopes (indicated by the dashed grey line). This is due to the fact that the $A_{\mathrm{RP}}$ simulations have more reaccretion due to recycling. One should correct the gas inflow in those simulations by a factor of $f_{\text {rec }}(\dot{M})=4.0$ due to this effect. The solid black lines are the predictions from the Neistein \& Dekel (2008) model (equation 2). The agreement between the data and the model is truly remarkable (taking $f_{\mathrm{rec}}(\dot{M})$ into account for $A_{\mathrm{RP}}$ ).

a couple of distinct peaks. These peaks can still be seen in the averaged data, especially if the number of galaxies in a certain bin is small (i.e. the MN simulation). Those peaks correspond to bigger clumps which are the merger events. We use the term 'merger' to describe any major or minor merger of mass ratio $\mu_{\mathrm{m}} \geq 0.1$, as distinct from 'smooth flows', which include 'miniminor' mergers with mass ratios $\mu_{\mathrm{m}}<0.1$. We will refer to this bimodality of the modes of inflow, smooth accretion versus clumps or merger events, throughout the whole paper.

The MN simulation is in general more noisy which can be attributed to its lower resolution. The $A_{\mathrm{RP}}$ simulation has a lower stellar and dark matter infall because of its much lower virial mass. The gas infall of the $A_{\mathrm{RP}}$ simulation on the other hand is higher than that of the ART or MN simulation, which is due to the fact that the $A_{\mathrm{RP}}$ simulation has stronger feedback than the ART suite by design. Additionally to all the feedback, the ART suite of simulation already has, it also includes feedback by radiation pressure. Ceverino et al. (2014) compared the effects of the feedback models used in ART and $A_{\mathrm{RP}}$ suites of simulations. SFR and stellar masses were lower by a factor $\sim 3$ with the stronger feedback. Zolotov et al. (2015) computed the outflow rates (their figs 2 and 3) of the $A_{\mathrm{RP}}$ suite and they found outflow rates similar or even higher than the SFR. Therefore the $A_{\mathrm{RP}}$ suite has more massive outflows and part of the outflowing material can rain down back to the central galaxy. An effect usually coined 'recycling' (Oppenheimer et al. 2010). As we will see later in Fig. 3, the gas inflow in the $A_{\mathrm{RP}}$ suite of sim- ulations is enhanced due to recycling compared to the MN or the ART simulations. The inflow of the $A_{\mathrm{RP}}$ simulations is higher by a factor $f_{\text {rec }}(\dot{M})=4.0$ compared to the MN or the ART simulations due to this effect. This factor for the inflow stays constant over the whole redshift range and also over the whole host halo mass range considered. We will also see a similar effect of recycling later in the paper during the fitting procedure of the distributions.

Otherwise all three suites behave similar: the gas inflow is roughly constant at around $100 \mathrm{M}_{\odot} \mathrm{yr}^{-1}$. Teklu (2012) reports a similar behaviour in SPH simulations. She finds a total gas inflow $\dot{M}=90 \mathrm{M}_{\odot} \mathrm{yr}^{-1}$ for a galaxy with $M_{\mathrm{vir}}=1.5 \times 10^{12} \mathrm{M}_{\odot}$ at $z=2.33$. Coming back to our own analysis and Fig. 2, we see that the MN simulation seems to have a marginally higher averaged gas inflow than the ART which can be accounted for by the 16 per cent more mass the average $\mathrm{MN}$ galaxy has. The gas inflow of the $A_{\mathrm{RP}}$ simulation in turn is even higher, which can be accounted for by either recycling or more efficient cooling from more efficient metal enrichment.

In $\mathrm{MN}$ we see that there is less stellar infall, but more gas infall by roughly a factor of $\sim 2$ compared to the ART simulations. In $A_{\mathrm{RP}}$ there is even less stellar infall and even more gas infall. The stellar inflow behaviour in general is extremely noisy. Its inflow rates oscillate between a few and up to about $80 \mathrm{M}_{\odot} \mathrm{yr}^{-1}$ for both, with the ART and the $A_{\mathrm{RP}}$ being more constant compared to $\mathrm{MN}$, which is due to the fact that the bins of the ART and the $A_{\mathrm{RP}}$ simulations consists of considerably more galaxies than the 
bins of the MN (see Table 1). Any peaks in the inflow profile (e.g. due to merger events) are much more smoothed out by simply the averaging process. It is noteworthy that in the stellar distribution there is only signal of inflow at the positions of the very peaks of the gas inflow, indicating that the smooth stream inflow component is fairly depleted from stars. The stars seem to flow into the central galaxy in the form of bigger clumps only. It is in this sense that the stars seem to follow the positions of the gas as we will refer to later. The lines for the dark matter inflow behave similar at larger radii. They exhibit slightly decreasing infall between $400 \mathrm{M}_{\odot} \mathrm{yr}^{-1}$ at $1.7 r_{\text {vir }}$ down to $200 \mathrm{M}_{\odot} \mathrm{yr}^{-1}$ at $0.7 r_{\text {vir }}$. Inside of $\sim 0.7 r_{\text {vir }}$ the three suites differ: $\mathrm{MN}$ stays constant although the scatter is larger, whereas ART and $A_{\mathrm{RP}}$ continue to decrease. The ART decreases down to $50 \mathrm{M}_{\odot} \mathrm{yr}^{-1}$ at $0.2 r_{\text {vir }}$ and the $A_{\mathrm{RP}}$ down to $50 \mathrm{M}_{\odot} \mathrm{yr}^{-1}$ already at $0.8 r_{\text {vir }}$.

The averaged amount of inflow into the galaxies as a function of radius is by and large constant with radius $r$ in all of the massredshift bins. This does not come as a surprise since the inflowing mass should be roughly conserved. The following systematic variations of the amount of inflow as a function of host halo mass $M_{\mathrm{vir}}$ and redshift $z$ become apparent. (a) The averaged amount of inflow increases with increasing host halo mass. (b) Both, the amount of inflow as well as the halo mass increase by roughly the same factor (one order of magnitude higher halo mass results in roughly one order of magnitude higher inflow) and (c) the total amount of inflow decreases slightly with increasing time (decreasing redshift). These trends are most convincingly seen in gas.

In the following we will make frequent use of basic statistical measures of our samples, namely the linearly averaged mean $\langle\dot{M}\rangle$, the mean of the logarithm $\left(\mu_{0}\right)$ and the standard deviation $\left(\sigma_{0}\right)$. The reader should note that those (unlike the later defined $\mu_{1}, \mu_{2}$, $\sigma_{1}, \sigma_{2}$ or $a_{1 / 2}$ ) are intrinsic properties of the distributions, that are independent from any model, equation or fits to it. Their definitions are:

$$
\begin{aligned}
\langle\dot{M}\rangle & =\frac{1}{n_{\mathrm{s}}} \sum_{\mathrm{i}=1}^{\mathrm{n}_{\mathrm{s}}} \dot{M}_{\mathrm{i}} \\
\mu_{0} & =\frac{1}{n_{\mathrm{s}}} \sum_{\mathrm{i}=1}^{\mathrm{n}_{\mathrm{s}}} \log _{10}\left(\dot{M}_{\mathrm{i}}\right) \\
\sigma_{0} & =\sqrt{\frac{1}{n_{\mathrm{s}}} \sum_{\mathrm{i}=1}^{\mathrm{n}_{\mathrm{s}}}\left[\log _{10}\left(\dot{M}_{\mathrm{i}}\right)-\mu_{0}\right]^{2}} .
\end{aligned}
$$

$n_{\mathrm{s}}$ is the total number of shells in the sample, so the number of galaxies in the respective bin times the number of different radii which are used. $\dot{M}_{\mathrm{i}}$ is the amount of gas inflow through the respective shell.

The theoretical prediction (Neistein \& Dekel 2008) of the average gas inflow rate $\langle\dot{M}\rangle$ into a host halo as a function of virial mass $M_{\text {vir }}$ and redshift $z$ is given by

$$
\langle\dot{M}\rangle=100 \frac{\mathrm{M}_{\odot}}{\mathrm{yr}}\left(\frac{1+z}{3.5}\right)^{2.5}\left(\frac{M_{\mathrm{vir}}}{10^{12} \mathrm{M}_{\odot}}\right)^{1.15} .
$$

To compare our results to this prediction, the magnitude of the inflow in the simulations will be averaged over the whole radius range for all mass-redshift bins. The resulting averaged inflow values $M_{\text {inflow }}$ of the gas are shown in Fig. 3 as a function of halo mass $M_{\text {vir }}$ and redshift $z$. In order to match $z=2.46$ or $M_{\text {vir }}=10^{12} \mathrm{M}_{\odot}$ in Fig. 3 the data points had to be rescaled according to the above equation (i.e. the data in the left-hand panel was rescaled according to $\langle\dot{M}\rangle \propto[1+z]^{2.5}$ and the data in the right-hand panel was rescaled according to $\langle\dot{M}\rangle \propto\left[M_{\mathrm{vir}}\right]^{1.15}$ ). The original values of redshift (lefthand panel) or host halo mass (right-hand panel) are given in colour coding. Equation (2) is overlain as solid black lines in both panels.

The simulated values for the inflow of the MN and the ART simulations are consistent with the theoretical predictions. The agreement is robust over three orders of magnitude in halo mass and also over the entire redshift range. The simulated inflow values of $A_{\mathrm{RP}}$ on the other hand are higher than the other two suites of simulations and the theoretical prediction. Interestingly, the $A_{\mathrm{RP}}$ suite clearly follows the same slopes with respect to both, the redshift and the halo mass, still. This parallel shift by a constant factor $f_{\text {rec }}(\dot{M})=4.0$ is due to the fact that the $A_{\mathrm{RP}}$ simulations have more massive outflows and therefore more reaccretion due to recycling. Since equation (2) describes only the primary accretion via cold streams and not the secondary accretion due to recycling, the $A_{\mathrm{RP}}$ simulations should be scaled down by this factor. We will also see a similar effect of recycling later in the paper during the fitting procedure of the distributions.

Accounting for recycling in the way described leads to an excellent agreement between the theoretical prediction and all the simulations including the $A_{\mathrm{RP}}$ suite, too. Only the highest redshift/lowest mass bins of both the MN and the ART suite of simulation lie a little bit further away from the relation. This is most probably due to the fact that these two bins are in a point in parameter space where the simulations have a lower resolution and might therefore be more vulnerable to numerical effects.

Gas supply of high mass galaxies $\left(M_{\mathrm{vir}}>10^{12} \mathrm{M}_{\odot}\right)$ is theoretically predicted (Dekel \& Birnboim 2006) to be quenched at low redshifts $(z<2)$. It would be nice to spot this effect in simulations. However, this turns out to be a difficult task to do: the ART galaxies are all at roughly $M_{\mathrm{vir}} \sim 10^{12} \mathrm{M}_{\odot}$, the $A_{\mathrm{RP}}$ galaxies are even lighter and both are therefore too light to test this prediction. Neither the $10^{12} \mathrm{M}_{\odot}$ nor the $5 \times 10^{12} \mathrm{M}_{\odot}$ nor the $10^{13} \mathrm{M}_{\odot}$ bins of the MN simulations (Table 1) show drops of the gas infall from $z=2.46$ down to $z=1.57$ (see the values presented in Fig. 3) that exceed the statistical uncertainties (compare the $1 \sigma$ standard deviations shown in Fig. 2). Unfortunately the simulations do not produce galaxies in such high-mass regimes at any earlier epochs sufficiently frequent enough. More high-resolution simulations of those high sigma peak galaxies are needed to establish a possible quenching of gas supply at low redshifts for high-mass haloes in simulations. However, such simulations would be computationally extremely demanding.

\section{DISTRIBUTIONS}

In Section 3, the average amount of inflow was presented. In this section, the behaviour of the underlying distributions is discussed as a function of host halo mass and redshift. So the likelihoods of certain deviations from the means presented in Section 3 are determined. For this the amount of inflow is measured through thin spherical shells at a number (200) of different radii between 0.4 and $1.7 r_{\text {vir }}$ around all available galaxies separately. This is done for every redshift and mass bin from all three suites of simulations. Having collected all these data, no average as in Section 3 is computed, but instead histograms of how often a certain inflow value occurs are compiled. All measurements of the amount of inflow are treated equal, regardless of the radius they were obtained at. The resulting normalized distributions of the gas inflow are plotted in Fig. 4 for all three suites of simulations (MN, ART as well as $A_{\mathrm{RP}}$ ) as filled blue histograms. Similar figures showing the distributions of the baryonic inflow instead of the gas were produced, found to 


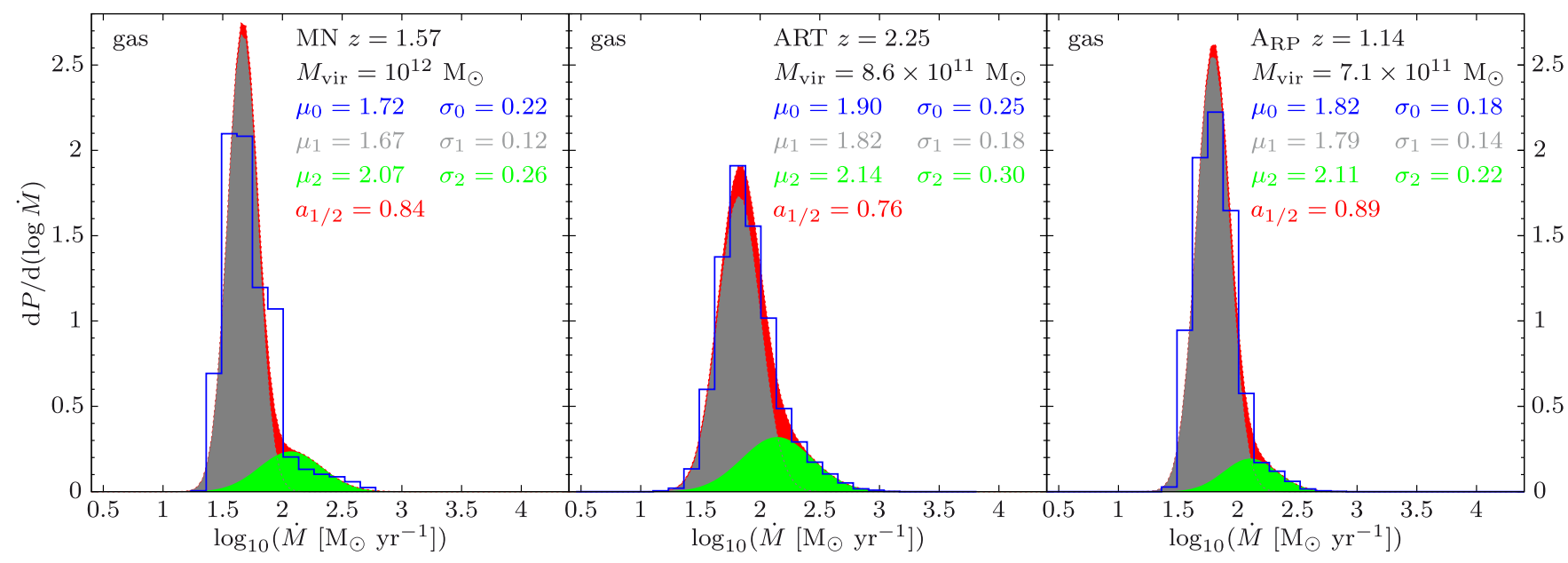

Figure 4. Normalized distributions of the gas inflow of selected mass-redshift bins for MN (left-hand panel), ART (centre panel) and $A_{\mathrm{RP}}$ (right-hand panel) in the radius range from 0.4-1.7 $r_{\text {vir }}$. The filled blue histograms are the real distributions from the simulations. Shown in red are the individual double-Gaussian (equation 3) fits to that particular panel. A double Gaussian consists of the sum of a first Gaussian (plotted in grey) and a second Gaussian (plotted in green). $\mu_{0}$ and $\sigma_{0}$ denote the statistical measures of the real underlying distribution, whereas $\mu_{1}, \mu_{2}, \sigma_{1}, \sigma_{2}$ and $a_{1 / 2}$ from equation (3) are the best-fitting parameters for the double Gaussian. In all of these, panels there is again a very beautiful double-Gaussian behaviour, the quality of the fits is quite striking in all three panels. It is most impressive for the right-hand panel, since the $A_{\mathrm{RP}}$ suite is our technically most advanced simulation.

look extremely similar and therefore omitted. Some trends are seen, most striking: the overall mean $\left(\mu_{0}\right.$, as defined in equation 1$)$ of the sample distributions (examples are shown in Fig. 4 as filled blue histograms) appears to increase with increasing mass and also with increasing redshift.

The best description for those histograms is the sum of a strong first Gaussian (plotted in grey) with a smaller mean $\left(\mu_{1}\right)$ and an also smaller standard deviation $\left(\sigma_{1}\right)$ and a weaker second Gaussian (plotted in green) having a bigger mean $\left(\mu_{2}\right)$ and a bigger standard deviation $\left(\sigma_{2}\right)$. This sum (plotted in red) can be expressed as the following 'double-Gaussian' distribution:

$$
\begin{aligned}
f(x)= & \frac{a_{1 / 2}}{\sigma_{1} \sqrt{2 \pi}} \exp \left[\frac{-\left(x-\mu_{1}\right)^{2}}{2 \sigma_{1}^{2}}\right] \\
& +\frac{1-a_{1 / 2}}{\sigma_{2} \sqrt{2 \pi}} \exp \left[\frac{-\left(x-\mu_{2}\right)^{2}}{2 \sigma_{2}^{2}}\right]
\end{aligned}
$$

The variable $a_{1 / 2}$ gives the fractional strength of the first to the second Gaussian. This model reflects the two different physical origins of the inflow: the majority of the gas inflow is expected to flow into the host halo by smooth accretion and a much smaller amount is expected to enter the host by merger events. Each of these mechanisms is accounted for by one of the two Gaussians of the distribution. Inflow by smooth accretion will naturally occur with a lower amount of inflow at any one instant (small $\mu_{1}$ ) but contribute much more to the inflow (big $a_{1 / 2}$ ) whereas inflow by merger events will have a much higher amount of inflow at any one instant (big $\mu_{2}$ ) but contributes much less to the inflow (small $\left.1-a_{1 / 2}\right)$. So the first Gaussian of this distribution represents the infall by smooth accretion whereas the second Gaussian of this distribution represents the infall by merger events.

It is useful to introduce the double Gaussian functional form at this point for several reasons: first, because it is a neat and very accurate description of the behaviour of the inflow distribution in hydrodynamical simulations. Secondly, it reflects nicely the physics of the bimodal nature of the inflowing material (mergers versus smooth inflow). Last but not least, this functional form's parameters teach us directly the mean accretion rate for the mergers $\left(\mu_{2}\right)$ or for the smooth component $\left(\mu_{1}\right)$, their standard deviations $\left(\sigma_{2}\right.$, $\sigma_{1}$ ) and maybe most importantly the relative strength of accretion via the smooth component compared to the accretion via merger events $\left(a_{1 / 2}\right)$. The above formula has already been used to describe the shape of the specific star formation rate distribution at redshifts $z \leq 2$ in observational work (Sargent et al. 2012, their equation 1). The similarity indicates that smooth accretion might fuel main-sequence star formation on the one hand and accretion through merger events might fuel star formation in starburst events on the other hand.

The overall equation (shown in red) is an excellent description for all three suites of simulations. Only exceptions are the very lowmass $\left(M_{\mathrm{vir}}=10^{11} \mathrm{M}_{\odot}\right) \mathrm{MN}$ galaxies which are noisy and the low redshift $(z \leq 1.7)$ ART cases in which either the second Gaussian (green) is tiny or its mean $\left(\mu_{2}\right)$ is not much bigger than the mean $\left(\mu_{1}\right)$ of the first Gaussian (grey). The double-Gaussian behaviour can most beautifully been seen in the technically most advanced $A_{\mathrm{RP}}$ suite of simulations. Here, all bins show an almost prototypical behaviour in that sense.

We now want to look at the characteristics of the model. Therefore, we fit the distributions (examples are plotted as filled blue histograms in Fig. 4) with equation (3) using $\mu_{1}, \mu_{2}, \sigma_{1}, \sigma_{2}$ and $a_{1 / 2}$ as free parameters. We do this for all mass-redshift bins given in Table 1. At this point we are seeking the best fit for each bin individually. Examples of the resulting fits are overplotted in Fig. 4 as filled red areas. The values of the parameters for all of those fits are presented in Fig. 5 as a function of halo mass $M_{\mathrm{vir}}$ and redshift $z$. An analogue plot for the baryons was also produced, since it looks very similar to the gas plot it was omitted. The plots show that $\mu_{1}$ as well as $\mu_{2}$ increase with increasing redshift, they also increase with increasing halo mass. A physical interpretation of these trends is that there is more inflow into heavier haloes and that there is also more inflow at earlier cosmic times. The $A_{\mathrm{RP}}$ values for $\mu_{1}$ and $\mu_{2}$ are considerably higher than their ART or MN counterparts. This is an effect of recycling. For the $\sigma_{1}$ or $\sigma_{2}$ values the data are noisy and no clear trends are visible. Some of the values for $a_{1 / 2}$ are also noisy. For gas however one can say that in more than three quarter of all the cases $(17 / 22) a_{1 / 2}$ is above 0.75 . The mean value for $a_{1 / 2}$ 


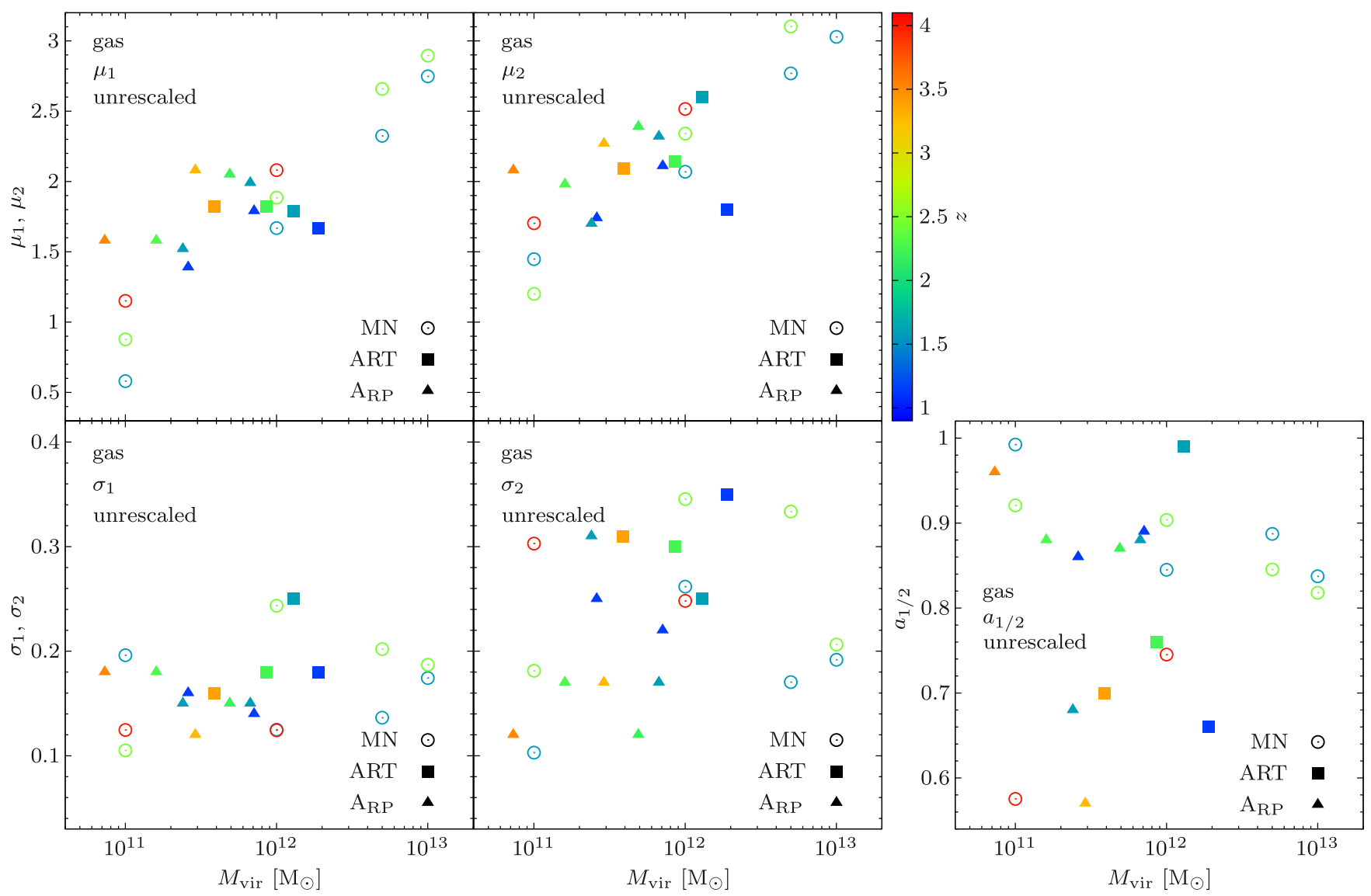

Figure 5. Results of the fits from equation (3) to the gas distributions of all of the bins mentioned in Table 1, examples are plotted in Fig. 4. Shown are the variations of the five parameters $\mu_{1}, \mu_{2}, \sigma_{1}, \sigma_{2}$ and $a_{1 / 2}$ from equation (3) as a function of halo mass $M_{\mathrm{vir}}$ and redshift $z$. These are the parameters of a fit. In this plot, the data are completely unrescaled, unlike the same data shown in Fig. 6 . All $A_{\mathrm{RP}}$ values for $\mu_{1}$ and $\mu_{2}$ are due to effects of recycling higher than expected. Physically these parameters show that (a) there is more absolute inflow into heavier haloes, (b) there is more absolute inflow at earlier cosmic times and there are heavier mergers happening (c) at earlier epochs and also for (d) higher mass host haloes. An analogue plot for the baryons was also produced, since it looks very similar to this one it was omitted.

averaged over all 22 mass-redshift bins from the three different suites is $\left\langle a_{1 / 2}\right\rangle=0.82$. These two numbers indicate according to the physical interpretation of the double-Gaussian curve that at least three quarter (possibly rather four-fifth) of the inflowing material is coming in via smooth streams and only less than one quarter (rather one-fifth) is coming in via merger events. This is in agreement with the findings of Romano-Díaz et al. (2014) who demonstrated that galaxies grow predominantly by smooth accretion from cosmological filaments.

From the values shown in Fig. 5 we deduce that the mean accretion rate for the mergers (given by $\mu_{2}$ ) is larger than that of the smooth component (given by $\mu_{1}$ ). In $\log$ space, it is higher by $\sim 0.5$ which means that it is roughly a factor 2-3 larger. These values indicate in the terms of galaxy formation that significantly more material is coming into the host halo via smooth accretion than via merger events. The standard deviation of the merger accretion rate $\left(\sigma_{2}\right)$ is $0.2-0.3$ dex, showing no trend with mass or redshift. For the smooth component $\left(\sigma_{1}\right)$ it is $0.12-0.24 \mathrm{dex}$. There are only marginal differences between gas and baryons. The gas seems to account for the majority of the mass of the baryons. We calculated the gas fraction from the simulations and find that $f_{\text {gas }}=85$ per cent. This is the case for all three suites of simulations, MN, ART as well as $A_{\mathrm{RP}}$.

We want to have a more general model and found that the behaviour with mass and redshift of two out of the five parameters, namely of $\mu_{1}$ and of $\mu_{2}$ can best be described by a logarithmic relation:

$\boldsymbol{X}=\boldsymbol{A}_{X} \ln (z+1)+\boldsymbol{B}_{X} \ln \left(\frac{M_{\mathrm{vir}}}{\mathrm{M}_{\odot}}\right)+\boldsymbol{C}_{X}$.

It is valid for the two parameters $\mu_{1}$ and $\mu_{2}$, denoted by $\boldsymbol{X}$, whereas $\boldsymbol{A}_{\boldsymbol{X}}, \boldsymbol{B}_{X}$ and $\boldsymbol{C}_{\boldsymbol{X}}$ are the three free parameters describing the logarithmic relation for both parameters. When fitting this equation, we had to account for the very strong signs of recycling found in the $A_{\mathrm{RP}}$ suite of simulations, as we already did for Fig. 3: there we showed the amount of inflow and found that the values for the $A_{\mathrm{RP}}$ simulations had to be scaled down by some factor due to the effects of rescaling. In the following we are going to fit two of the parameters of equation (3) which are also prone to be affected by recycling. The positions of the centres of the first and the second Gaussian of the distribution on the inflow ( $\mu_{1}$ and $\mu_{2}$ as defined by equation 3 ) in the $A_{\mathrm{RP}}$ simulations are considerably higher than in the $\mathrm{MN}$ or the ART simulations. However, the reader should note that those are completely different physical values than the average amount of inflow $\langle\dot{M}\rangle$ which are related but their relation is nonlinear and therefore the effect of recycling has a differently strong effect on each of them. i.e. we have to scale the values for $\langle\dot{M}\rangle$, $\mu_{1}$ and $\mu_{2}$ for the $A_{\mathrm{RP}}$ simulations by independent scaling factors. The $\mu_{1}$ values of the $A_{\mathrm{RP}}$ simulation are a factor of $f_{\text {rec }}\left(\mu_{1}\right)=1.45$ higher than the corresponding values for $\mu_{1}$ of the MN or the ART 
Table 2. The best-fitting values for two $\left(\mu_{1}\right.$ and $\left.\mu_{2}\right)$ out of the five parameters of equation (3) when fitted with our model (equation 4 ) to the data of all bins as given in Table 1 as shown in in examples in Fig. 4 (red filled areas and labels). The other three parameters $\left(\sigma_{1}, \sigma_{2}\right.$ and $\left.a_{1 / 2}\right)$ were far too noisy to allow any meaningful fits. The column $f_{\text {frec }}\left(\mu_{1 / 2}\right)$ indicates the factor the $A_{\mathrm{RP}}$ suite of simulations was scaled down by to account for its strong recycling. The actual comparison of the model (equation 4) using the tabulated values and the simulations can be seen in Fig. 6, the accuracy is exceptional.

\begin{tabular}{ccccc}
\hline $\boldsymbol{X}$ & $\boldsymbol{A}_{\boldsymbol{X}}$ & $\boldsymbol{B}_{\boldsymbol{X}}$ & $\boldsymbol{C}_{\boldsymbol{X}}$ & $f_{\mathrm{rec}}\left(\mu_{1 / 2}\right)$ \\
\hline$\mu_{\text {1gas }}$ & 0.87 & 0.44 & -11.4 & 1.45 \\
$\mu_{\text {2gas }}$ & 0.79 & 0.39 & -9.4 & 1.20 \\
\hline
\end{tabular}

simulations. The $\mu_{2}$ values of the $A_{\mathrm{RP}}$ simulation are a factor of $f_{\text {rec }}\left(\mu_{2}\right)=1.20$ higher than the corresponding values for $\mu_{2}$ of the MN or the ART simulations. Again both factors remain constant over the whole redshift and host halo mass range considered. We present the best-fitting values for $\boldsymbol{A}_{\boldsymbol{X}}, \boldsymbol{B}_{\boldsymbol{X}}, \boldsymbol{C}_{\boldsymbol{X}}$ and $f_{\mathrm{rec}}\left(\mu_{1 / 2}\right)$ in Table 2.

In Fig. 6, we compare the general model of equation (4) with the parameters of the double Gaussians of the simulated distributions. Show are the positions of the centres of the first ( $\mu_{1}$, upper panels) and the second ( $\mu_{2}$, lower panels) Gaussian of the distributions of the inflow as a function of mass (left-hand panels) and as a function of redshift (right-hand panels). There are very strong logarithmic dependences present in all panels as described by our general model which is overlain as solid black lines. The data points within each panel were scaled with the help of the scaling relation in the corresponding other panel to the values indicated. The colour bar axes indicate the values the data points used to have before rescaling. Please refer to Fig. 5 for the unrescaled data. The panels show that our simple phenomenological model (equation 4 and Table 2) is an excellent description of the data.

\section{CONCLUSIONS}

In this paper, we looked at the inflow rates of accretion along streams from the cosmic web into galaxies at high redshifts using three sets of AMR hydrocosmological simulations. We calculated the amount of inflow as a function of radius, host halo mass and redshift. We computed the distribution of inflow rates and we found the following.

(i) The inflow rates are roughly constant with radius, their behaviour with host halo mass and redshift follows clearly the predictions of Neistein \& Dekel (2008).

(ii) The distributions of the $\log$ accretion rates can be very well described by a 'double-Gaussian' functional form (equation 3 ) that is the sum of two Gaussians, the primary corresponding to 'smooth' inflow and the secondary to 'mergers'.

(iii) Most of the amount of inflow ( $>80$ per cent) is entering the halo at low inflow rates (i.e. smooth accretion) and only a small portion $(<20$ percent) of the inflow is coming in at high inflow rates (massive merger events).

(iv) Two out of the five parameters of the double-Gaussian function (namely $\mu_{1}$ and $\mu_{2}$ ) have a strong redshift as well as host halo mass dependence. In equation (4), Table 2 and Fig. 6, we present a simple phenomenological model, which describes the shape of the double-Gaussian distribution as a function of mass and redshift. (v) The standard deviation of the total accretion rate is 0.2 0.3 dex, showing no trend with mass or redshift.

(vi) The suite of simulations that include strong feedback shows massive signs of accretion due to recycling: i.e. for the $A_{\mathrm{RP}}$ simulations we see (compared to the MN or ART simulations that have only weak feedback) an increase in the amount of inflow by a factor $\sim 4.0$, independent of mass and redshift. Due to the same effect an increase in $\mu_{1}$ of the order of $\sim 1.45$ and an increase in $\mu_{2}$ of the order of $\sim 1.2$ (see table 2 ) is also seen.

(vii) There are hardly any differences between the gas and the baryons. Gas seems to account for the majority of the mass of the baryons anyway and additionally the star formation seem to locally follow the gas.

(viii) There is more absolute inflow into heavier haloes compared to lighter haloes also there is more absolute inflow at earlier cosmic times compared to later cosmic times.

A double-Gaussian distribution has already been found by Sargent et al. (2012), who looked at the distributions of specific star formation rates at fixed stellar mass in observations. They interpreted the bimodality of this functional form as contributions from either main-sequence star formation or star formation during starburst activity. The analogy between their and our findings indicates that connections might exist between smooth accretion and main-sequence star formation on the one hand as well as accretion through merger events and starburst activity star formation on the other hand. However, the reader should note that just having the same functional form is no proof.

In our most advanced simulations, the $A_{\mathrm{RP}}$ simulations, which include the strongest feedback recipes, we see strong evidence for the reaccretion of formerly ejected material. This suite of simulations has more massive outflows and part of the outflowing material rains back again to the central galaxy. An effect coined 'recycling' (Oppenheimer et al. 2010). The inflow in the $A_{\mathrm{RP}}$ suite of simulations is enhanced due to recycling compared to the MN or the ART simulations. This effect provides a third distinct accretion mode along with the cold and hot modes described by Kereš et al. (2005) and Dekel \& Birnboim (2006).

One potential limitation of our simulations may arise from the artificial pressure floor imposed in order to properly resolve the Jeans mass. This may have an effect on the temperature and density of the densest and coldest parts of the streams, with potential implications on the estimated inflow rates. Still modern hydrodynamical simulation codes are the best available tools for recovering the stream properties. With $17-70 \mathrm{pc}$ resolution, and with proper cooling below $10^{4} \mathrm{~K}$, these simulations provide the most reliable description of the cold streams so far.

In a forthcoming companion paper (Goerdt et al., in preparation), we are going to address the role of mergers versus smooth flows by analysing the clumpiness of the gas streams. We evaluate each clump mass and estimate a mass ratio for the expected merger. Finally, we will look at the distribution of constituents amongst the clumps.

We conclude that gas is flowing into a galaxy's halo mainly as smooth accretion flows with only a minority ( $<25$ per cent) coming in via merger events such as clumps or small satellite galaxies. The accretion rate distributions can be described by a double-Gaussian decomposition (equation 3 ). That this functional form is also used to describe the specific star formation rate distributions in observations could indicate that it is the smooth accretion that fuels main-sequence star formation on the one hand and the accretion 


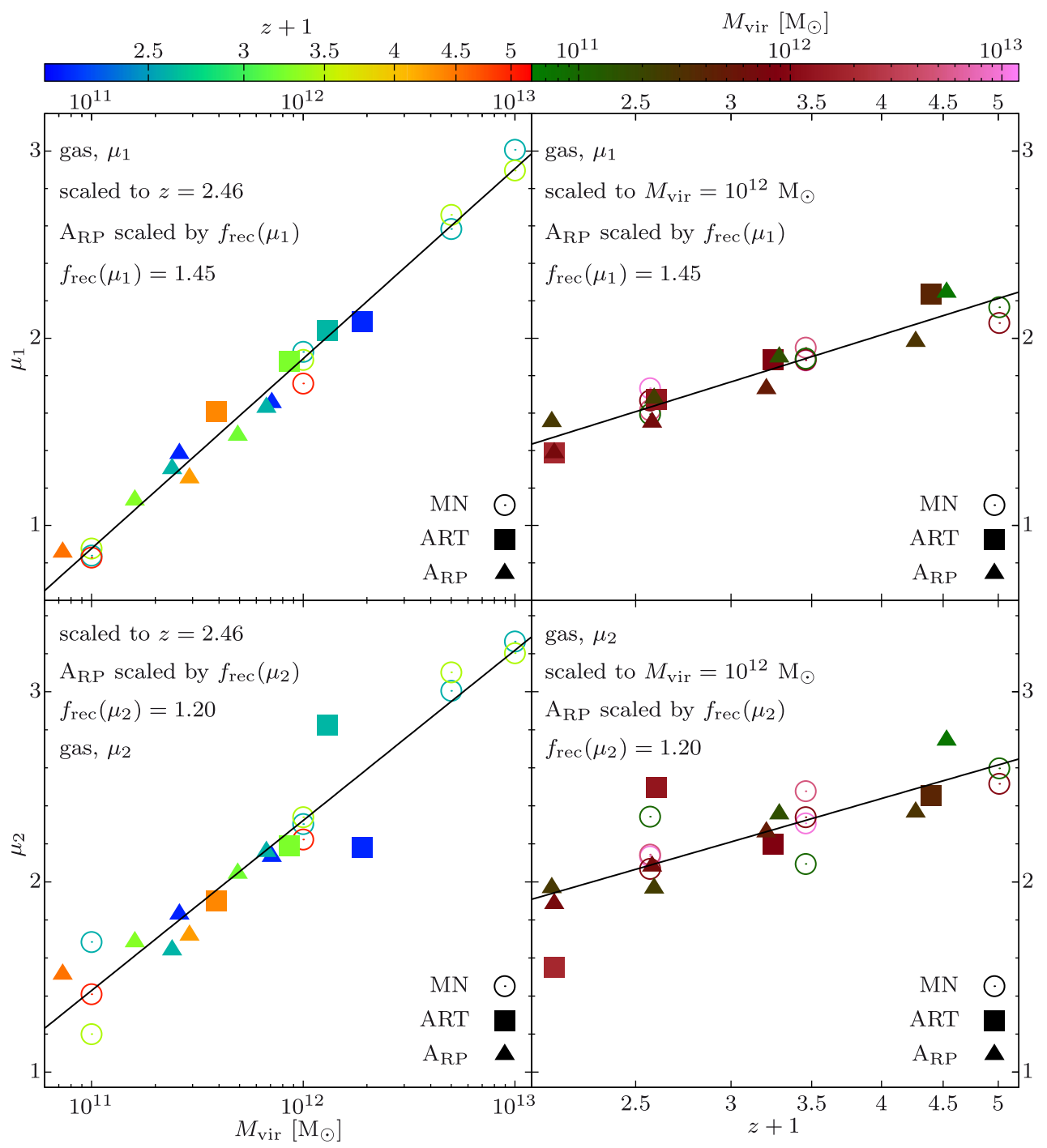

Figure 6. Shown are the positions of the centres of the first ( $\mu_{1}$, upper panels) and the second ( $\mu_{2}$, lower panels) Gaussians of the distributions of the inflow as a function of mass (left-hand panels) or as a function of redshift (right-hand panels). There are very strong logarithmic dependences present in all panels as described by equation (4) which is overlain as solid black lines. The data points within the left-hand panels were scaled with the help of the scaling relation in the corresponding right-hand panel to the values indicated. The colour bar axes indicate the values the data points used to have before rescaling. Please refer to Fig. 5 for the unrescaled data. The panels show that our simple phenomenological model (equation 4 and Table 2) is an excellent description of the data.

of material through merger events on the other hand that fuels star formation of starburst events.

\section{ACKNOWLEDGEMENTS}

TG is a Lise Meitner fellow. We thank the anonymous referee for useful comments which greatly aided the clarity of this work. We acknowledge stimulating discussions with Nicolas Bouché, Oliver Czoske, Natalia Romero and Mark Sargent. TG would like to thank the University Observatory, Munich where parts of this work where carried out for their hospitality. The simulations were performed in the astro cluster at HU, at the National Energy Research Scientific Computing Center (NERSC), Lawrence Berkeley National Laboratory and at NASA Ames Research Center. Parts of the computational calculations were done at the Vienna Scientific Cluster under project number 70522. This work was supported by FWF project number M 1590-N27 and by MINECO project number AYA 2012-32295.

\section{REFERENCES}

Agertz O., Teyssier R., Moore B., 2011, MNRAS, 410, 1391

Agertz O., Kravtsov A. V., Leitner S. N., Gnedin N. Y., 2013, ApJ, 770, 25

Almeida J. S., Elmegreen B. G., Muñoz-Tuñón C., Elmegreen D. M., 2014, A\&AR, 22, 71

Behroozi P. S., Wechsler R. H., Conroy C., 2013, ApJ, 770, 57

Birnboim Y., Dekel A., 2003, MNRAS, 345, 349

Bouché N. et al., 2010, ApJ, 718, 1001

Cen R., 2014, ApJ, 789, L21

Ceverino D., Klypin A. A., 2009, ApJ, 695, 292

Ceverino D., Dekel A., Bournaud F., 2010, MNRAS, 404, 2151

Ceverino D., Dekel A., Mandelker N., Bournaud F., Burkert A., Genzel R., Primack J., 2012, MNRAS, 420, 3490

Ceverino D., Klypin A., Klimek E. S., Trujillo-Gomez S., Churchill C. W., Primack J., Dekel A., 2014, MNRAS, 442, 1545

Ceverino D., Dekel A., Tweed D., Primack J., 2015, MNRAS, 447, 3291

Ceverino-Rodriguez D., 2008, PhD thesis, New Mexico State University

Daddi E. et al., 2010, ApJ, 713, 686

Dalla Vecchia C., Schaye J., 2008, MNRAS, 387, 1431 
Dekel A., Birnboin Y., 2006, MNRAS, 368, 2

Dekel A. et al., 2009, Nature, 457, 451

Dekel A., Zolotov A., Tweed D., Cacciato M., Ceverino D., Primack J. R., 2013, MNRAS, 435, 999

Dubois Y., Teyssier R., 2008, A\&A, 477, 79

Fardal M. A., Katz N., Gardner J. P., Hernquist L., Weinberg D. H., Davé R., 2001, ApJ, 562, 605

Faucher-Giguère C., Keres D., Ma C., 2011, MNRAS, 417, 2982

Ferland G. J., Korista K. T., Verner D. A., Ferguson J. W., Kingdon J. B., Verner E. M., 1998, PASP, 110, 761

Förster Schreiber N. M. et al., 2009, ApJ, 706, 1364

Förster Schreiber N. M. et al., 2011, The Messenger, 145, 39

Fumagalli M., Prochaska J. X., Kasen D., Dekel A., Ceverino D., Primack J. R., 2011, MNRAS, 418, 1796

Genel S. et al., 2008, ApJ, 688, 789

Genel S., Bouché N., Naab T., Sternberg A., Genzel R., 2010, ApJ, 719, 229

Genzel R. et al., 2008, ApJ, 687, 59

Genzel R. et al., 2010, MNRAS, 407, 2091

Goerdt T., Ceverino D., 2015, MNRAS, 450, 3359

Goerdt T., Dekel A., Sternberg A., Ceverino D., Teyssier R., Primack J. R., 2010, MNRAS, 407, 613

Goerdt T., Dekel A., Sternberg A., Gnat O., Ceverino D., 2012, MNRAS, 424, 2292

Goerdt T., Burkert A., Ceverino D., 2013, preprint (arXiv:1307.2102)

Grevesse N., Sauval A. J., 1998, Space Sci. Rev., 85, 161

Haardt F., Madau P., 1996, ApJ, 461, 20

Ibata R. A. et al., 2013, Nature, 493, 62

Katz N., Hernquist L., Weinberg D. H., 1992, ApJ, 399, 109

Kennicutt R. C., 1998, ApJ, 498, 541

Kereš D., Katz N., Weinberg D. H., Davé R., 2005, MNRAS, 363, 2

Kereš D., Katz N., Fardal M., Davé R., Weinberg D., 2009, MNRAS, 395, 160

Komatsu E. et al., 2009, ApJS, 180, 330

Kravtsov A. V., 2003, ApJ, 590, 1

Kravtsov A. V., Klypin A. A., Khokhlov A. M., 1997, ApJS, 111, 73

Lacey C., Cole S., 1993, MNRAS, 262, 627

Miller G. E., Scalo J. M., 1979, ApJS, 41, 513
Moody C. E., Guo Y., Mandelker N., Ceverino D., Mozena M., Koo D. C., Dekel A., Primack J., 2014, MNRAS, 444, 1389

Moster B. P., Naab T., White S. D. M., 2013, MNRAS, 428, 3121

Neistein E., Dekel A., 2008, MNRAS, 388, 1792

Neistein E., van den Bosch F. C., Dekel A., 2006, MNRAS, 372, 933

Nelson D., Genel S., Pillepich A., Vogelsberger M., Springel V., Hernquist L., 2015, preprint (arXiv:1503.02665)

Ocvirk P., Pichon C., Teyssier R., 2008, MNRAS, 390, 1326

Oppenheimer B. D., Davé R., Kereš D., Fardal M., Katz N., Kollmeier J. A., Weinberg D. H., 2010, MNRAS, 406, 2325

Rees M. J., Ostriker J. P., 1977, MNRAS, 179, 541

Robertson B. E., Kravtsov A. V., 2008, ApJ, 680, 1083

Rodighiero G. et al., 2011, ApJ, 739, L40

Romano-Díaz E., Shlosman I., Choi J., Sadoun R., 2014, ApJ, 790, L32

Sargent M. T., Béthermin M., Daddi E., Elbaz D., 2012, ApJ, 747, L31

Schaye J., Dalla Vecchia C., 2008, MNRAS, 383, 1210

Silk J., 1977, ApJ, 211, 638

Springel V., Hernquist L., 2003, MNRAS, 339, 289

Teklu A., 2012, Bachelor thesis, LM University, Munich

Teyssier R., 2002, A\&A, 385, 337

Truelove J. K., Klein R. I., McKee C. F., Holliman J. H., Howell L. H., Greenough J. A., 1997, ApJ, 489, 179

van de Voort F., Schaye J., 2012, MNRAS, 423, 2991

van de Voort F., Schaye J., Booth C. M., Haas M. R., Dalla Vecchia C., 2011, MNRAS, 414, 2458

Wetzel A. R., Nagai D., 2015, ApJ, 808, 40

White S. D. M., Rees M. J., 1978, MNRAS, 183, 341

Woods R. M., Wadsley J., Couchman H. M. P., Stinson G., Shen S., 2014, MNRAS, 442, 732

Woosley S. E., Weaver T. A., 1995, ApJS, 101, 181

Yepes G., Kates R., Khokhlov A., Klypin A., 1997, MNRAS, 284, 235

Zolotov A. et al., 2015, MNRAS, 450, 2327

This paper has been typeset from a $\mathrm{TE}_{\mathrm{E}} \mathrm{X} / \mathrm{L} \mathrm{T} \mathrm{E}$ file prepared by the author. 